\title{
The Model of Reflective Surface Based on the Scattering Layer with Diffuse Substrate and Randomly Rough Fresnel Boundary
}

\author{
V.P. Budak ${ }^{1}$, A.V. Grimailo ${ }^{1}$ \\ BudakVP@gmail.com|GrimailoAV@gmail.com \\ ${ }^{1}$ National Research University "Moscow Power Engineering Institute", Moscow, Russia.
}

In this article, we describe the mathematical model of the reflective surface as a scattering layer with the diffuse substrate and randomly rough Fresnel boundary. This model opens the way for a physically correct description of the light reflection processes with polarization account and hence enables engineers and designers to obtain much more precise results in their work. The algorithm of Fresnel boundary modeling based on the method of mathematical expectations reduces calculation time by constructing the randomly rough surface only at the ray trajectory nodes instead of constructing realizations of a random field. As a part of the complete reflective surface model, the algorithm made it able for us to model the effect of the average lens emergence.

Keywords: mathematical model, reflection, refraction, polarization, reflective surface, light scattering.

\section{Introduction}

Nowadays it is a tradition for light engineering that the light polarization state is not considered when modeling light distribution. This neglection is acceptable when we deal with diffusely reflecting surfaces and a small number of re-reflections. On the contrary, we must account the influence of light polarization when considering surfaces with a significant specular part. The very first reflection changes the state of light polarization and this fact affects all the following processes of light distribution.

To date, a series of proceedings devoted to the light polarization account has been published [3, 6, 7]. Basing on use of ray tracing and local estimations of the Monte-Carlo Method they show that accounting of the light polarization state leads to quite significant changes not only in the qualitative results but in the quantitative results as well.

However, the mathematical model of multiple reflections with polarization account used for estimating the influence of polarization showed just the first approximation for the quantitative results. Therefore, the following step of the model development is to create and use the physically correct model of the reflective surface.

We must consider that the light is always reflected from both of the faces of the material surface and the material volume. The light penetrates the near-surface layers of the material where the light scattering by the material particles occurs. Then, a certain fraction of the initial luminous flux re-enters the surrounding space. At this point, the role of polarization account takes an exceedingly significant part as it influences all the processes of the light scattering.

Thus, the authors decided to develop the model of reflective surface, which would account the effects described above. Further, the physically correct model will enable us to obtain more precise results of light distribution modeling.

\section{Mathematical model of the reflective surface}

When the light penetrates the near-surface layers, the processes occurring have the same nature as the radiative transfer in turbid media. Additionally, we must account that a real material surface is always uneven owing to the most varied causes (corpuscular structure of the material, surface treatment defects, etc.) and never reflects the light according to only specular or only diffuse law but there take place both of them at the same time.

Thus, we decided to represent the reflective surface as a scattering layer with a diffusely reflecting plane at the bottom and randomly rough Fresnel boundary above (Fig. 1).

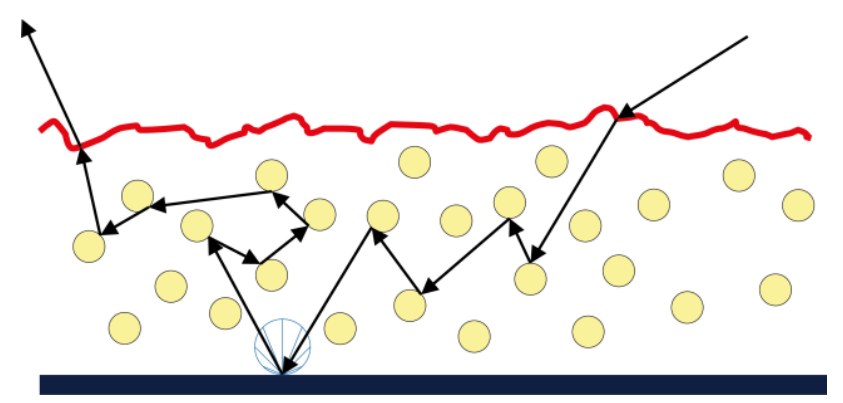

Fig 1. Representation of the reflective surface in the mathematical model.

Generally, scattering media are characterized by matrix scatter coefficient $\vec{\sigma}$, matrix absorption coefficient $\vec{\kappa}$ and matrix extinction coefficient $\vec{\varepsilon}=\vec{\kappa}+\vec{\sigma}$. Neglection of dichroism, birefringence, and similar effects of the same kind, which are inherent only for several materials, enables us to transit to the scalar analogs of the matrix coefficients $\varepsilon=\kappa+\sigma$.

For modeling the reflective surface with the assumptions taken above, we need to solve the boundary value problem for the vector radiative transfer equation. Let us consider the planeparallel system of the scattering layer with a diffuse substrate. The layer is irradiated at the angle $\theta$ by the plane monodirectional source with random polarization state. Then one can write the problem as

$$
\left\{\begin{array}{l}
\left.\mu \frac{\partial}{\partial \tau} \mathbf{L}(\tau, \hat{\mathbf{l}})+\mathbf{L}(\tau, \hat{\mathbf{l}})=\frac{\Lambda}{4 \pi} f\right] \overrightarrow{\mathrm{R}}\left(\hat{\mathbf{l}} \times \hat{\mathbf{l}}^{\prime} \rightarrow \hat{\mathbf{N}} \times \hat{\mathbf{l}}\right) \times \\
\times \vec{x}\left(\tau, \hat{\mathbf{l}}, \hat{\mathbf{l}}^{\prime}\right) \overrightarrow{\mathbf{R}}\left(\hat{\mathbf{N}} \times \hat{\mathbf{l}}^{\prime} \rightarrow \hat{\mathbf{l}} \times \hat{\mathbf{l}}^{\prime}\right) \mathbf{L}(\tau, \hat{\mathbf{l}}) d \hat{\mathbf{l}}^{\prime}, \\
\mathbf{L}(0, \mu>0, \varphi)=\mathbf{L} \delta\left(\hat{\mathbf{l}}-\hat{\mathbf{l}}^{\prime}\right), \\
\mathbf{L}(\tau, \mu \leq 0, \varphi)=[\rho E / \pi \quad 0 \quad 0 \quad 0]^{\mathrm{T}},
\end{array}\right.
$$

where $\hat{\mathbf{I}}$ and $\hat{\mathbf{l}}^{\prime}$ are the unit vectors of the scattered and incident ray directions respectively;

$\hat{\mathbf{N}}$ is the normal vector;

$\mathbf{L}$ is radiance (Stokes vector);

$\mu=\cos \theta$;

$\tau=\int_{z_{0}}^{z_{1}} \varepsilon(z) d z$ is the optical track thickness in the section

$\left[z_{0}, z_{1}\right]$ 
$\Lambda=\frac{\sigma}{\varepsilon}$ is the single scatter albedo;

$\vec{x}\left(\tau, \hat{\mathbf{l}}, \hat{\mathbf{l}}^{\prime}\right)$ is the scatter matrix;

$\overrightarrow{\mathbf{R}}\left(\hat{\mathbf{l}} \times \hat{\mathbf{l}}^{\prime} \rightarrow \hat{\mathbf{N}} \times \hat{\mathbf{l}}\right)$ is the matrix of the reference plane rotation from $\hat{\mathbf{l}} \times \hat{\mathbf{l}}^{\prime}$ to $\hat{\mathbf{N}} \times \hat{\mathbf{l}}$.

\section{Randomly rough Fresnel boundary}

One of the most important components of the reflective surface model described above is the construction of the randomly rough Fresnel boundary. To show the importance of taking into account properties of a randomly rough surface one can give the cases of observing ocean currents, underwater mountain ranges, and shoals by people from outer space.

For the first time, the deep-sea bottom topography from space was observed by American astronaut Gordon Cooper from the Gemini 5 spacecraft in August 1965. The first of the Soviet cosmonauts were A. G. Nikolaev and V. I. Sevastyanov from the Soyuz-9 spacecraft in June 1970. At the same time, they first drew attention to the fact that the sea waves, ripples on its surface are not an obstacle when observing the topography of the seabed from space.

Then there was a series of other known observations through the rough surface of the ocean:

1. August 1974. G. V. Sarafanov and L. S. Demin observed the bottom relief at depth of hundreds of meters from the Soyuz-15 spacecraft. They succeeded to see the bottom of the Mozambique Gulf that separates the island of Madagascar from the African continent. The cosmonauts saw a bottom, covered with shafts that stretch along the strait. The structure of the strait bottom resembles the structure of that of a small river, but the dimensions are many times larger than in the river.

2. June 1975. From the board of the Salyut-4 orbital station, P. I. Klimuk and V. I. Sevastyanov observed the bottom of the seas and oceans. When flying over the Atlantic Ocean from Newfoundland to the Canary Islands, they clearly saw ocean currents. Along the European coast of the Mediterranean Sea with an emerald strip of subtropical greenery, they saw under the water a continuation of the continent relief. Continuation of the relief was also visible on the eastern coast of South America - three terraces extending deep into the Atlantic Ocean. It was visible how far the Amazon River carried its muddy waters into the ocean, how they were carried away by deep currents under a layer of clean water.

3. June 1978. Underwater relief of the Pacific Ocean bottom in the region of the Solomon Islands at depths of up to 400 meters was observed by V.V. Kovalenok and A. S. Ivanchenkov. During the flight, the cosmonauts first made an attempt to derive the laws of the most favorable conditions for observing underwater formations. These observations were carried out from an orbit close to the solar one at a small height of the Sun above the horizon.

Experience shows that the best conditions for observation are when the height of the Sun above the horizon is $30^{\circ}-60^{\circ}$; direction from the Sun $90^{\circ}-130^{\circ}$; viewing angles do not exceed $30^{\circ}-40^{\circ}$ from the direction of the nadir and, of course, outside the glare zone.

In the cases described above, the so-called statistical lens effect appeared due to a randomly rough Fresnel surface at the ocean-atmosphere boundary. This effect allowed cosmonauts and astronauts to observe the bottom of the seas and oceans from outer space at great depths.

The problem of the randomly rough Fresnel boundary modeling is unavoidably encountered in the solution of a large number of physical problems in various fields. In the majority of practical cases, the shape of a randomly rough surface is described by a random function of coordinates (and sometimes of time). Therefore, scattering on a real surface should be considered as a statistical task, which consists in finding the probabilistic characteristics of a scattered field from known statistical characteristics of the random surface. The methods of solving such a problem are the same regardless of the physical nature of the roughness [1].

Thus, researchers of the radiative transfer processes in the ocean-atmosphere system face a similar task when describing the effect of the perturbed sea surface on the radiation field.

There are two ways for solving this problem [4]. In the first one, realizations of a random field $\zeta$ are constructed according to the randomization principle, then one simulates random trajectories $\hat{\mathbf{I}}$ and on their basis calculate the random estimates of the sought-for functionals. The main difficulty of employing this approach is the necessity to find the intersection points of the ray and surface at each trajectory node. In the general case, the determination of the intersection coordinates costs much computational time.

The second approach is preferable, therefore. It is based on the method of mathematical expectations (Fig. 2). Here, to construct an $N$-component random trajectory we need to have the realizations of the random surface only at $N$ points, calculated in a certain way [4]. At the points of rays reaching a random interface, the selection is made of random realizations of normals to the surface.

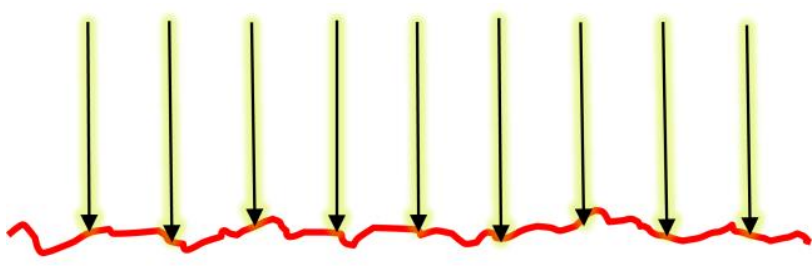

Fig 2. To the second approach of the randomly rough Fresnel boundary modeling.

\section{Algorithm of Fresnel boundary modeling}

In order to make clear the way one can use the second approach from the latter section, let us consider [4] an arbitrary trajectory of $n$ nodes

$$
\left\{\left(\mathbf{r}_{0}, \hat{\mathbf{l}}_{0}, \mathbf{Q}_{0}\right),\left(\mathbf{r}_{1}, \hat{\mathbf{l}}_{1}, \mathbf{Q}_{1}\right), \ldots,\left(\mathbf{r}_{n}, \hat{\mathbf{l}}_{n}, \mathbf{Q}_{n}\right)\right\}
$$

where $\mathbf{r}_{i}$ is the $i$-th ray collision point on the surface or in the medium;

$\hat{\mathbf{l}}_{i}$ is a unit vector of the ray direction after the $i$-th collision;

$\mathbf{Q}_{i}$ is the vector weight after $i$-th collision (its components correspond to those of the Stokes vector).

A set of the random surface point corresponds to the nodes of the trajectory:

$$
\left\{\left(\zeta\left(\mathbf{r}_{\perp, 1}\right), \hat{\mathbf{N}}\left(\mathbf{r}_{\perp, 1}\right)\right), \ldots,\left(\zeta\left(\mathbf{r}_{\perp, n}\right), \hat{\mathbf{N}}\left(\mathbf{r}_{\perp, n}\right)\right)\right\},
$$

where

$$
\begin{aligned}
& \mathbf{r}_{\perp}=(x, y) \text { are horizontal coordinates so that } \mathbf{r}=\left(\mathbf{r}_{\perp}, z\right) ; \\
& \zeta\left(\mathbf{r}_{\perp}\right) \text { is the random surface roughness function; } \\
& \hat{\mathbf{N}}\left(\mathbf{r}_{\perp}\right) \text { is the outer normal to the surface at the point } \\
& \mathbf{r}=\left(\mathbf{r}_{\perp}, \zeta\left(\mathbf{r}_{\perp}\right)\right) .
\end{aligned}
$$


At the first step, one samples a random value of deviation $\zeta_{1}$ from the interval $\left(-h_{m}, h_{m}\right)$ according to the probability density based on the normal distribution. Then one evaluates the distance $\xi$ from the point $\mathbf{r}_{0}$ to the plane $z=\zeta\left(\mathbf{r}_{\perp}\right)$ in the direction $\hat{\mathbf{I}}_{0}$. The coordinates of the first trajectory node are evaluated by using the formula

$$
\mathbf{r}_{1}=\mathbf{r}_{0}+\xi \hat{\mathbf{l}}_{0}
$$

Thus, the point $\mathbf{r}_{1}$ is the first point of the ray intersection with the random surface, provided that there have been no intersections before. We allow for this condition by multiplying the vector weight $\mathbf{Q}_{0}$ by the probability $P\left(\mathbf{r}_{0}, \mathbf{r}_{1}\right)$ for the ray $\mathbf{r}_{1}=\mathbf{r}_{0}+\xi \hat{\mathbf{l}}_{0}$ to have no intersections with the surface on the track between the points $\mathbf{r}_{0}$ and $\mathbf{r}_{1}$.

The last statement requires some clarification. In general case, instead of $P\left(\mathbf{r}_{0}, \mathbf{r}_{1}\right)$, one needs to use another probability $P\left(\mathbf{r}_{0}, \mathbf{r}_{1} \mid \zeta_{1}\right)$, the expression for which has the following form:

$$
P\left(\mathbf{r}_{i-1}, \mathbf{r} \mid \zeta_{i-1}, \ldots, \zeta_{1}\right)=\int \Delta\left(\mathbf{r}_{i-1}, \mathbf{r}, \zeta\right) d P(\zeta)
$$

where $\zeta=\left(\zeta, \zeta_{x}, \zeta_{y}\right): \quad \zeta \in\left[-h_{m}, h_{m}\right], \quad \zeta_{x}, \zeta_{y} \in(-\infty,+\infty)$; $\zeta, \zeta_{x}, \zeta_{y}$ are the random functions possessing normal one-dimensional distributions with the parameters $\left(0, \sigma_{\zeta}\right),\left(0, \sigma_{x}\right)$ and $\left(0, \sigma_{y}\right)$ respectively;

$\Delta\left(\mathbf{r}, \mathbf{r}^{\prime}\right)=\left\{\begin{array}{ll}1, & \left|\mathbf{r}^{\prime}-\mathbf{r}\right| \leq \xi_{0}, \\ 0 & \left|\mathbf{r}^{\prime}-\mathbf{r}\right| \leq \xi_{0}\end{array} ;\right.$

$\xi_{0}$ is the minimal distance from the point $\mathbf{r}$ to the medium boundary in the direction $\hat{\mathbf{l}}$.

The standard deviations $\sigma_{x}$ and $\sigma_{y}$ are not independent and connected with the standard deviation $\sigma_{\zeta}$ of the random quantity $\zeta$ by the following expression

$$
\sigma_{x}=\sigma_{y}=\sigma_{\zeta} \sqrt{\left|K_{\zeta}^{\prime \prime}(0)\right|},
$$

where $K_{\zeta}=K_{\zeta}\left(\Delta \mathbf{r}_{\perp}\right)$ is the correlation function of deviations for isotropic undulation. In case of anisotropic undulations, we have to set functions $K_{\zeta, x}$ and $K_{\zeta, y}$. In applied calculations, functions of the form are often used as a correlation function:

$$
K_{\zeta}(\Delta \mathbf{r})=\exp \left\{-\left(\frac{\Delta \mathbf{r}}{\rho}\right)^{2}\right\}
$$

where $\rho$ (and $\sigma_{\zeta}$ ) are the parameters determining the force and shape of undulation.

Calculation of probabilities (5) is necessary on each step of modeling trajectory. Since the formula (5) is extremely complicated for direct calculation and practically inapplicable, the formulae obtained in [1] are often used when solving such problems provided that $P\left(\mathbf{r}_{i-1}, \mathbf{r}_{1} \mid \zeta_{i-1}, \ldots, \zeta_{1}\right)=P\left(\mathbf{r}_{i-1}, \mathbf{r}\right)$. These formulae can be applied in two extreme cases:

$$
\begin{aligned}
& \text { when } \frac{\sigma_{\zeta}}{\rho} \square 1 \\
& \qquad \begin{array}{l}
P\left(\mathbf{r}_{i-1}, \mathbf{r}_{i}\right)=\exp \left\{-\int_{0}^{t_{1}} K_{\zeta}(t) d t\right\}, \\
t_{1}=\left[z_{i}-h-\zeta_{i-1}\right]\left[\left(\hat{\mathbf{I}}_{i-2}, \mathbf{k}\right)^{2}-1\right], \quad \mathbf{k}=\left[\begin{array}{lll}
0 & 0 & 1
\end{array}\right],
\end{array}
\end{aligned}
$$

when $\frac{\sigma_{\zeta}}{\rho} \square 1$

$$
\begin{gathered}
P\left(\mathbf{r}_{i-1}, \mathbf{r}_{i}\right)=\Phi\left\{\frac{e}{\sigma_{\zeta} \sqrt{e^{2}-3}} \times\right. \\
\left.\times\left[\zeta_{i-1}\left(1-\frac{1}{e}\right)-\frac{\rho}{e} \sqrt{\frac{z_{x}^{2}\left(\mathbf{r}_{\perp, i-1}\right)+z_{y}^{2}\left(\mathbf{r}_{\perp, i-1}\right)}{2}}+\rho\left[\left(\hat{\mathbf{i}}_{i-2}, \mathbf{k}\right)^{2}-1\right]\right]\right\}, \\
\Phi(x)=\frac{1}{\sqrt{2 \pi}} \int_{-\infty}^{x} e^{-t^{2} / 2} d t .
\end{gathered}
$$

A random realization of the normal vector $\hat{\mathbf{N}}_{1}$ is sampled from the set of the unit vectors $\left\{\hat{\mathbf{N}}:\left(\hat{\mathbf{N}}, \hat{\mathbf{I}}_{0}\right)<0, \hat{\mathbf{N}} \in \Omega_{+}\right\}$by the following way. Using normal distribution, one model $z_{x}$ and $z_{y}$ with the distribution parameters $\left(0, \sigma_{x}\right)$ and $\left(0, \sigma_{y}\right)$ respectively.

The quantities $z_{x}$ and $z_{y}$ are substituted into the following formula and one evaluates the components of the vector $\hat{\mathbf{N}}$

$$
\begin{aligned}
& \hat{\mathbf{N}}\left(\mathbf{r}_{\perp}\right)=\frac{\mathbf{k}-\mathbf{e}\left(\mathbf{r}_{\perp}\right)}{\sqrt{1+\left|\mathbf{e}\left(\mathbf{r}_{\perp}\right)\right|^{2}}}, \\
& \mathbf{e}\left(\mathbf{r}_{\perp}\right)=\left[\begin{array}{lll}
z_{x} & z_{y} & 0
\end{array}\right] .
\end{aligned}
$$

Having obtained the values of $\hat{\mathbf{N}}_{1}$ and $\hat{\mathbf{l}}_{0}$, we are able to gain [5] the Fresnel reflection factor $R\left(\hat{\mathbf{l}}_{0}, \hat{\mathbf{N}}_{1}\right)$ :

$$
R(\hat{\mathbf{l}}, \hat{\mathbf{N}})=\frac{(|A|-B)^{2}\left(A^{2} B^{2}+C^{2}\right)}{(|A|+B)^{2}(|A| B+C)^{2}},
$$

$$
\begin{aligned}
& A=(\hat{\mathbf{l}}, \hat{\mathbf{N}}), B=\sqrt{1 / v^{2}-1+A^{2}}, \quad C=1-A^{2}, \\
& \gamma=\sqrt{1-v^{2}\left(1-A^{2}\right)}-v A, \\
& v= \begin{cases}1 / n, & (\hat{\mathbf{l}}, \hat{\mathbf{N}}) \leq 0, \\
n, & (\hat{\mathbf{l}}, \hat{\mathbf{N}})>0,\end{cases}
\end{aligned}
$$

where $n$ is the refractive index of the material with respect to air. We consider $R\left(\hat{\mathbf{I}}_{0}, \hat{\mathbf{N}}_{1}\right)$ as a probability for the ray $\hat{\mathbf{I}}_{0}$ having collided with the facet of normal $\hat{\mathbf{N}}_{1}$ to undergo the mirror reflection, and $1-R\left(\hat{\mathbf{I}}_{0}, \hat{\mathbf{N}}_{1}\right)$ as a probability for the ray to undergo refraction. 
In this way, the coefficient $R\left(\hat{\mathbf{I}}_{0}, \hat{\mathbf{N}}_{1}\right)$ is used to choose the type of interaction with the surface from two possible outcomes: reflection and refraction. Having made the choice, one defines the vector $\hat{\mathbf{l}}_{1}$ according to the formulae

$$
\hat{\mathbf{l}}_{1}= \begin{cases}\hat{\mathbf{l}}_{0}-2\left(\hat{\mathbf{l}}_{0}, \hat{\mathbf{N}}\right) \hat{\mathbf{N}}, & \text { for reflection, } \\ v \hat{\mathbf{l}}_{0}+\gamma \hat{\mathbf{N}}, & \text { for refraction. }\end{cases}
$$

After that, one can make the transformation of the vector weight according to the following expression

$\mathbf{Q}_{1}=\overrightarrow{\mathbf{R}}\left(\hat{\mathbf{l}}_{0} \times \hat{\mathbf{l}}_{1} \rightarrow \hat{\mathbf{N}}_{1} \times \hat{\mathbf{l}}\right) \ddot{\rho}\left(\mathbf{r}_{1}, \hat{\mathbf{l}}_{1}, \hat{\mathbf{l}}_{0}\right) \overrightarrow{\mathbf{R}}\left(\hat{\mathbf{N}}_{0} \times \hat{\mathbf{l}}_{0} \rightarrow \hat{\mathbf{l}}_{0} \times \hat{\mathbf{l}}_{1}\right) \mathbf{Q}_{0}$,

where $\overrightarrow{\mathbf{R}}\left(\hat{\mathbf{N}}_{0} \times \hat{\mathbf{l}}_{0} \rightarrow \hat{\mathbf{l}}_{0} \times \hat{\mathbf{l}}_{1}\right)$ is the matrix of the reference plane rotation from $\hat{\mathbf{N}}_{0} \times \hat{\mathbf{l}}_{0}$ to $\hat{\mathbf{l}}_{0} \times \hat{\mathbf{l}}_{1}$;

$\vec{\rho}\left(\mathbf{r}_{1}, \hat{\mathbf{l}}_{1}, \hat{\mathbf{l}}_{0}\right)$ is the Mueller matrix for reflection or refraction depending on the choice based on the coefficient $R\left(\hat{\mathbf{l}}_{0}, \hat{\mathbf{N}}_{1}\right)$.

The next steps of the algorithm have the same logic with the exception that we need to account light scattering in the material medium and thus, solve a rather complicated problem of estimating probabilities and evaluating ray weights.

\section{Conclusion}

At the current stage, the realization of the algorithm described above enabled us to model the effect of the average lens emergence. Further, we are going to use the model of the randomly rough Fresnel boundary as a part of the reflective surface model (Section 2).

Basing on the reflective surface representation as a scattering layer with the diffuse substrate and randomly rough Fresnel boundary above, we will be able to construct a complete model of reflection with the account of scattering in the material volume.

The main role in solving the problem (1) should be given to the analytical methods [2], as they are much faster than numerical those. This approach will pave the way for us to integrate the model into existing methods, used in computer graphics (e. g. ray tracing, photon maps, local estimations, etc.). It will enable engineers and designers to account polarization when solving practical tasks and thus obtain much more precise results of light distribution calculation and visualization.

Nevertheless, analytical methods always imply the use of certain assumptions, the effect of which on the result is currently possible to estimate only by using the Monte-Carlo Methods. In the future, it is interesting to compare the two variants of the mathematical model and, possibly, combine them, taking into account the advantages of each of the variants.

\section{References}

[1] Bass, F.G., Fuks, I.M. Wave Scattering from Statistically Rough Surfaces. Pergamon, 1979.

[2] Budak, V.P., Basov, A.Y. Modeling of a scattering slab with diffuse bottom and top reflecting by the Snell law. In Proceedings of GraphiCon 2018, p. 399-401.

[3] Budak, V.P., Grimailo, A.V. The influence of the light polarization account on the result of multiple reflections calculation. In Proceedings of GraphiCon 2018, p. 409-410.

[4] Kargin, B.A., Rakimgulov, K.B. A weighting Monte-Carlo method for modelling the optical radiation field in the oceanatmosphere system. Russ. J. Numer. Anal. Math. Modelling, Vol.7, No.3, pp. 221-240 (1992).
[5] Marchuk, G.I., et al. The Monte Carlo Methods in Atmospheric Optics. Springer-Verlag, Berlin, 1980.

[6] Mojzik, M., Skrivan, T., Wilkie, A., Krivanek, J. BiDirectional Polarised Light Transport. Eurographics Symposium on Rendering 2016.

[7] Wolff, L. B., Kurlander, D. J. Ray tracing with polarization parameter // IEEE Computer Graphics and Applications. 1990. V. 10, No. 6, P. 44-55. 